\title{
CLOSURE OF THE LINEAR SPAN OF AN EXPONENTIAL SYSTEM IN A WEIGHTED BANACH SPACE
}

\author{
ELIAS ZIKKOS
}

Abstract. For a certain class of sequences with repeated terms,

$$
\left\{\lambda_{n}, \mu_{n}\right\}_{n=1}^{\infty}:=\{\underbrace{\lambda_{1}, \lambda_{1}, \ldots, \lambda_{1}}_{\mu_{1} \text { times }}, \underbrace{\lambda_{2}, \lambda_{2}, \ldots, \lambda_{2}}_{\mu_{2} \text { times }}, \ldots, \underbrace{\lambda_{k}, \lambda_{k}, \ldots, \lambda_{k}}_{\mu_{k} \text { times }}, \ldots\},
$$

we prove that every function belonging to the closed span of the exponential system

$$
\left\{x^{k} e^{\lambda_{n} x}: n \in \mathbb{N}, k=0,1,2, \ldots, \mu_{n}-1\right\},
$$

in some weighted Banach spaces on the real line, extends analytically as an entire function by admitting a series representation of the form

$$
\sum_{n=1}^{\infty}\left(\sum_{k=0}^{\mu_{n}-1} c_{n, k} z^{k}\right) e^{\lambda_{n} z}, \quad c_{n, k} \in \mathbb{C}, \quad \forall z \in \mathbb{C} .
$$

Mathematics subject classification (2010): 30B50, 30B60, 46E15, 46E20.

Keywords and phrases: Completeness, closure, minimality, Taylor-Dirichlet series.

\section{REFERENCES}

[1] J. M. Anderson, K. G. Binmore, Closure theorems with applications to entire functions with gaps, Trans. Amer. Math. Soc. 161 (1971) 381-400.

[2] G. T. DENG, Incompleteness and closure of a linear span of exponential system in a weighted Banach space, J. Approx. Theory 125 no. 1 (2003), 1-9.

[3] W. H. J. Fuchs, On the closure of $\left\{e^{-t} t^{a_{n}}\right\}$, Proc. Cambridge Philos. Soc. 42 (1946), 91-105.

[4] P. Malliavin, Sur quelques procédés d'extrapolation, Acta Math. 93 (1955) 179-255.

[5] J. Ning, G. T. DenG, C. YI, Incompleteness and closure of the multiplicity system $\left\{t^{k} e^{\lambda_{j} t}\right\}$ in the weighted Banach space, J. Math. Anal. Appl. 341 no. 2 (2008), 1007-1017.

[6] X. YANG, Incompleteness of exponential system in the weighted Banach space, J. Approx. Theory $\mathbf{1 5 3}$ no. 1 (2008), 73-79.

[7] E. Zikkos, Completeness of an exponential system in weighted Banach spaces and closure of its linear span, J. Approx. Theory 146 no. 1 (2007), 115-148. 